\title{
EFEITO DO NÚMERO CUMULATIVO DE CIGARROS FUMADOS SOBRE O PERFIL LIPÍDICO E CLASSES DE FOSFOLIPÍDIOS DE UMA POPULAÇÃO DE CARUARU-PERNAMBUCO
}

\section{EFECTO DEL NÚMERO ACUMULADO DE CIGARRILLOS FUMADOS EN EL PERFIL LIPIDO Y LAS CLASES DE FOSLÍPIDO DE UNA POBLACÍON DE CARUARU-PERNAMBUCO}

\section{EFFECT OF CUMULATIVE NUMBER OF SMOKED CIGARRTES ON LIPID PROFILE AND PHOSPOLIPID CLASSES OF A CARUARU-PERNAMBUCO POPULATION}

\begin{abstract}
Antonio Lopes Ferreira Neto ${ }^{1}$; Isabela Simões Alves ${ }^{2}$; Jeaninne Alexandra de Azevedo Silva ${ }^{3}$; Ana Carolina Bezerra Paz ${ }^{4}$; Bianka Santana dos Santos ${ }^{5}$
\end{abstract}

DOI: https://doi.org/10.31692/978-65-991061-9-4.94-99

\section{INTRODUÇÃO}

O tabagismo tem significativa participação na fisiopatologia de Doenças

Cardiovasculares Ateroscleróticas (DCVAs), que perpassam por alterações nos metabolismos lipídicos e glicídicos dos indivíduos fumantes. Porém, não há na literatura muitos registros de correlação entre o número cumulativo de cigarros fumados e o aumento quantitativo do perfil lipídico (LEE et al., 2011). Tem-se, por outro lado, o conhecimento de que a nicotina induz a secreção de catecolaminas, cortisol e hormônio do crescimento $(\mathrm{GH})$, o que acarreta em um aumento na concentração sanguínea de ácidos graxos livres capazes de estimularem a secreção hepática de lipoproteína de muito baixa densidade (VLDL), triglicerídeos (TG) e lipoproteínas de baixa densidade (LDL), e de inibirem a secreção de HDL-C (LI et al., 2018). Contudo, são pouco conhecidos, os mecanismos de aumento dos níveis de fosfolipídios plasmáticos através do uso crônico de cigarro (RAMOS et al., 2004).

Dessa forma, o presente projeto desperta a atenção para o fato de que, se houver alteração nas subclasses de fosfolipídios plasmáticos, pode-se encontrar um elo para a possível redução dos níveis de HDL com o uso cumulativo do cigarro, acrescentando enormemente para uma explicação fisiopatológica da relação causa-consequência entre tabagismo e DCVAs.

\footnotetext{
${ }^{1}$ Acadêmico de Medicina, Núcleo de Ciências da Vida - Universidade Federal de Pernambuco - Campus do Agreste, antonio.lopes.neto@hotmail.com

2 Acadêmica de Medicina, Núcleo de Ciências da Vida - Universidade Federal de Pernambuco - Campus do Agreste.

${ }^{3}$ Acadêmica de Medicina, Núcleo de Ciências da Vida - Universidade Federal de Pernambuco - Campus do Agreste, jeaninne.med@gmail.com

4 Acadêmica de Medicina, Núcleo de Ciências da Vida - Universidade Federal de Pernambuco - Campus do Agreste, ana-carolina-bezerra-paz@hotmail.com

${ }^{5}$ Doutora em Ciências Biológicas e Pós-Doutora em Bioquímica e Fisiologia, Docente do Curso de Medicina, Núcleo de Ciências da Vida - Campus do Agreste - Universidade Federal de Pernambuco, biankasantana@gmail.com
} 
Ademais, a nicotina tem ação sobre o sistema nervoso central, promovendo a liberação de inúmeros neurotransmissores e, em decorrência disso, provocando dependência, que pode ser avaliada através do Teste de Fagerström (VAN AMSTERDAM et al., 2019). Assim sendo, este estudo tem como objetivo principal estudar o efeito do número cumulativo de cigarros sobre o metabolismo lipídico, e avaliar, em paralelo, o processo tolerância-dependência à nicotina e suas confluências para a saúde caruaruense.

\section{FUNDAMENTAÇÃO TEÓRICA}

As doenças cardiovasculares figuram entre as primeiras causas de mortalidade mundial, com destaque especial para a Doença Arterial Coronariana (DAC) e doenças neurovasculares, como o Acidente Vascular Encefálico (AVE), seja ele isquêmico ou hemorrágico. Nesse sentido, os principais fatores de risco para altos índices de morbimortalidade por DCVAs são as dislipidemias, o tabagismo e o etilismo (PRÉCOMA et al., 2019).

O fumo do tabaco implica em uma inalação muito grande de nicotina e outros poluentes, que são responsáveis por indução de estresse oxidativo e resposta inflamatória nos vasos sanguíneos, além de inibirem a ativação de substâncias antioxidantes (MUNAKATA et al., 2018). Destarte, o uso crônico de cigarro, e consequente de nicotina por fumantes correntes, induzem uma maior produção de radicais livres de oxigênio, os quais atuam inibindo a formação de óxido nítrico e aumentando a sua degradação, o que acarreta em uma menor distensão vascular e maior vasoconstricção (TODA; TODA, 2010). Por conseguinte, há aumento da resistência vascular periférica, e, quando nos rins, esse processo constritor, diminui a perfusão das arteríolas eferentes, aumentando concomitantemente a pressão arterial (MANGAN; STOTT; DHANDA, 2018).

Paralelamente, o hábito tabagista é claramente relacionado ao aumento considerável de LDL, que pode corroborar com um diagnóstico de dislipidemia. O tempo de início do uso crônico de cigarros, bem como a quantidade diária fumada, relacionam-se positivamente ao crescimento quantitativo e qualitativo de LDL, assim, aqueles que fumam cerca de 20 cigarros ao dia por mais de 15 anos podem ter riscos de dislipidemias muito maiores que aqueles que fumam essa quantidade ou menos entre 1 e 5 anos (SHI et al., 2018). Não obstante, a nicotina induz uma resistência insulínica através da indução de liberação do hormônio do crescimento e cortisol e também do aumento na quantidade de lipídios plasmáticos (MORGAN et al., 2004).

Ademais, o uso do teste de Fagerström para dependência de nicotina faz-se imprescindível para o entendimento do consumo diário de cigarros e da dependência química deste, bem como método preditivo para as taxas de tendência ao hábito de fumar. A avaliação 
do número cumulativo de cigarros fumados, por sua vez, também é importante, pois este número traz à tona os efeitos que o cigarro provocou em pessoas que já cessaram o hábito tabágico, ou seja, os ex-fumantes, além de evidenciar as alterações ocorridas nos fumantes correntes (VAN AMSTERDAM et al., 2019).

\section{METODOLOGIA}

Desenho do Estudo e Determinações Bioquímicas. Estudo transversal foi conduzido com 500 voluntários, com idade $\geq 18$ anos, de Caruaru-PE, após aprovação por Comitê de Ética em Pesquisa ( $n^{\circ}$ CAAE 54688716.6.0000.5208). Após 12h em jejum, amostras sanguíneas foram obtidas para determinação de Colesterol Total, Triglicerídios, LDL-colesterol e HDLcolesterol, por metodologias enzimáticas, e dos fosfolipídios por cromatografia de camada delgada (FOLCH et al., 1957). Número Cumulativo de Cigarros Fumados e Dependência à Nicotina. Foram acessados, respectivamente, conforme Brinkman \& Coates (1963) e pelo Teste de Fagerström. Análise Estatística dos Dados. Teste de correlação e t de Student $(p<0,05)$.

\section{RESULTADOS E DISCUSSÕES}

Foi encontrada uma prevalência de $13 \%$ de fumantes correntes (FC) e de $14 \%$ de exfumantes (EF) na população deste estudo. Malta et al. (2010) verificaram uma prevalência de 16,1\% de tabagismo no Brasil e dados da jPNS (2013) e do MINISTÉRIO DA SAÚDE BRASIL (2017) mostraram uma prevalência de 15\% no Estado de Pernambuco. Outrossim, o presente estudo também mostrou que a maior parte de FC e EF foi de fumantes severos, seguidos por fumantes considerados moderados e, por fim, fumantes leves. Brinkman e Coates (1963) já mostravam a importância desta estratificação, quando criaram o Índice de Brinkman. O valor médio encontrado do Índice de Brinkman no presente estudo foi de 977,8 \pm EPM de 327,5 , evidenciando a maior presença de fumantes severos nessas pessoas de Caruaru-PE. O número cumulativo de cigarros fumados correlacionou-se positivamente, de maneira significativa, com os níveis séricos de Colesterol Total (CT), LDL-colesterol (LDL-C), Triglicerídeos (TG) e Glicose, conforme demonstrado na Tabela 1. FC tiveram significativamente menores níveis plasmáticos de Lisofosfatidilcolina e maiores níveis de Fosfatidilcolina do que Não Fumantes (NF), sem, no entanto, diferirem quanto às outras classes de fosfolipídios, como mostrado na Tabela 2. Dependência à nicotina também foi encontrada e classificada como grave. 
Tabela 1. Correlação (r) de Pearson entre valores de Índice de Brinkman, glicemia e níveis de lipídios séricos.

\begin{tabular}{ccc}
\hline $\begin{array}{c}\text { Parâmetros } \\
\text { Avaliados }\end{array}$ & $\mathbf{r}$ & $* \mathbf{p}$ \\
\hline TG (mg/dL) & 0,371 & $<0,001$ \\
HDL-C (mg/dL) & 0,402 & $<0,001$ \\
CT (mg/dL) & 0,425 & $<0,001$ \\
LDL-C (mg/dL) & $-0,432$ & $<0,001$ \\
Glicose (mg/dL) & 0,337 & $<0,001$ \\
\hline
\end{tabular}

\begin{tabular}{ccc}
\multicolumn{3}{c}{ Tabela 2. Valores de Fosfolipídios Plasmáticos em Fumantes e Não Fumantes de Caruaru-PE. } \\
\hline $\begin{array}{c}\text { Fosfolipídios }(\boldsymbol{\mu m o l} \text { de } \\
\text { Fósforo/mL) }\end{array}$ & $\begin{array}{c}\text { Média } \mathbf{E} \text { EP } \\
\text { NF }\end{array}$ & $\begin{array}{c}\text { Média } \pm \mathbf{E P} \\
\text { FC }\end{array}$ \\
\hline *Fosfatidilcolina $(\mathbf{P C})$ & $1,959 \pm 0,02$ & $3,014 \pm 0,02$ \\
& $0,635 \pm 0,01$ & $0,2141 \pm 0,02$ \\
*Lisofosfatidilcolina (LPC) & $0,355 \pm 0,2$ & $0,365 \pm 0,03$ \\
*Fosfatidiletanolamina (PE) & $0,404 \pm 0,01$ & $0,418 \pm 0,03$ \\
*Esfingomielina (SPH)
\end{tabular}

Teste $t$ de Student desemparelhado. Os valores estão expressos em média \pm EP (Erro Padrão da Média). *Valores de p (nível de significância) encontrados entre FC versus NF foram $<0,05$. **Valores de p (nível de significância) encontrados entre FC versus NF não foram significativos.

Destarte, ratifica-se uma forte correlação entre Colesterol Total e LDL-colesterol e o tempo de uso do cigarro. Shi et al. (2018) também encontraram uma forte correlação entre o tempo de tabagismo, os níveis de Triglicerídios e de Colesterol Total em Chineses, ocorrendo, por conseguinte, redução nos níveis de LDL-C. Kakamu et al. (2013) têm identificado um número cumulativo de cigarros fumados superior a 600 , como foi a média encontrada neste estudo $(977,8)$, como um marcador efetivo de risco para diabetes mellitus. Isto vem a somar com o alto dano que o tabaco pode ocasionar ao metabolismo lipídico e, consequentemente, ampliar o risco de doenças cardiovasculares. Os dados do presente estudo evidenciam uma possível menor esterificação do colesterol na HDL e, portanto, uma possível redução no transporte reverso do colesterol, que somado aos menores níveis de HDL-C, pode levar a uma maior probabilidade de DCVAs, como reportado por Shi et al. (2018). Kirchenchtejn e Chatkin (2004) ressaltaram a necessidade de se investigar não apenas o número de cigarros, mas também o processo de tolerância-dependência à nicotina, pois o estado de dependência é dinâmico e não seria justo classificar as pessoas apenas como dependentes ou não. Précoma et al. (2019) 
também relataram que o tabagismo é uma causa primária de DCVAs e é extremamente importante distinguir entre os fumantes o grau de dependência à nicotina. O presente estudo encontrou uma dependência à nicotina considerada como grave, com escore igual a 8. Isto mostra o quanto a população de Caruaru, Agreste de Pernambuco, pode estar susceptível a doenças crônicas não transmissíveis decorrentes do hábito tabagista e o quão difícil pode ser para aqueles que fazem uso do cigarro de forma corrente cessarem este hábito.

\section{CONCLUSÕES}

O tabagismo é uma condição prevalente em Caruaru, apesar da redução em nível nacional, com uma relação de dependência à nicotina considerada grave, e que está diretamente associado com a presença de condições biológicas de maior risco cardiovascular nesta população, em especial, correlacionando-se com alterações relevantes no metabolismo lipídico, e no sistema circulatório, propiciando não apenas dislipidemias quantitativas, mas também alterações qualitativas de extrema relevância, como o possível prejuízo no transporte reverso do colesterol realizado pela HDL, sugerido pela diminuição de lisofosfatidilcolina e elevação de fosfatidilcolina encontrados no estudo. Por isso, faz-se improtelável aplicação de esforços na redução do estilo de vida tabagista da população caruaruense, bem como faz-se imprescindível utilização dos princípios de prevenção à saúde da atenção primária em saúde para construir conhecimento popular sobre os benefícios de parar de fumar.

\section{REFERÊNCIAS}

BRASIL. Vigitel Brasil 2016 : vigilância de fatores de risco e proteção para doenças crônicas por inquérito telefônico. Brasília: Ministério da Saúde, 2017.

BRINKMAN, G. L.; COATES, JR., E. O. The Effect of Bronchitis, Smoking, and Occupation on Ventilation. The American Review of Respiratory Disease, [s.i], v. 87, p. 684-693. 1963.

DING, Ning et al. Cigarette smoking, smoking cessation, and long-term risk of 3 major atherosclerotic diseases. Journal of the American College of Cardiology, [s.i], v. 74, n. 4, p. 498-507, 2019.

FOLCH, J.; LEES, M.; STANLEY, G. H. S. A simple mehod for the isolation and purification of total lipides from animal tissues. The Journal Of Biological Chemistry, Boston, p. 497509. 23 ago. 1957.

KIRCHENCHTEJN, C.; CHATKIN, J. M. Dependência da nicotina. Jornal Brasileiro de Pneumologia. São Paulo, v. 30, n. 2, p. S11-S18, ago. 2004.

LI, X. X. et al. Effects of smoking and alcohol consumption on lipid profile in male adults in northwest rural China. Public health, [s.i], v. 157, p. 7-13, 2018. 
MANGAN, Clodagh; STOTT, Martyn C.; DHANDA, Raman. Renal physiology: blood flow, glomerular filtration and plasma clearance. Anaesthesia \& Intensive Care Medicine, v. 19, n. 5, p. 254-257, 2018.

MORGAN, Thomas M. et al. Acute effects of nicotine on serum glucose insulin growth hormone and cortisol in healthy smokers. Metabolism, [s.i[, v. 53, n. 5, p. 578-582, 2004.

MUNAKATA, Satoru et al. Oxidative stress responses in human bronchial epithelial cells exposed to cigarette smoke and vapor from tobacco-and nicotine-containing

PRÉCOMA, D. B. et al. (Orgs.), Sociedade Brasileira de Cardiologia. Atualização da Diretriz de Prevenção Cardiovascular da Sociedade Brasileira de Cardiologia. Arquivos Brasileiros de Cardiologia, [s.i], v. 113, n. 4, p.787-891, 2019.

SHI, Jikang et al. Classified status of smoking and quitting has different associations with dyslipidemia in residents in northeast China. Clinica Chimica Acta, [s.i], v. 486, p. 209-213, 2018.

TODA, Noboru; TODA, Hiroshi. Nitric oxide-mediated blood flow regulation as affected by smoking and nicotine. European journal of pharmacology, [s.i], v. 649, n. 1-3, p. 1-13, 2010.

VAN AMSTERDAM, Jan et al. Use of the Fagerström test to assess differences in the degree of nicotine dependence in smokers from five ethnic groups: The HELIUS study. Drug and alcohol dependence, [s.i], v. 194, p. 197-204, 2019. 